\title{
Estado Nutricional de Idosos Residentes em Instituição Geriátrica e a Relação com o Consumo Alimentar
}

\author{
Ana Carolina Soares Rosa, ${ }^{1}$ Jéssica Ribeiro Roque, ${ }^{1}$ Danielle Raquel Gonçalves ${ }^{2}$
}

\begin{abstract}
RESUMO
O trabalho teve como objetivo avaliar o estado nutricional e o consumo alimentar de idosos institucionalizados, verificando-se possíveis relações entre eles. Tratou-se de um estudo transversal descritivo com abordagem quantitativa envolvendo 48 idosos. Foi avaliado peso (P), altura (A), índice de massa corporal (IMC), circunferências do braço (CB), panturrilha (CP) e cintura (CC), dobra cutânea do tríceps (DCT), e circunferência muscular do braço (CMB). O recordatório alimentar de 24 horas de três dias (R24h) foi utilizado para análise do consumo alimentar, sendo aplicado aos cuidadores da instituição. A correlação de Spearman foi aplicada para verificar a relação entre a antropometria e o consumo alimentar. A partir deste levantamento, foi encontrado que a maioria dos idosos estava com sobrepeso e adiposidade abdominal segundo IMC e CC, mas apresentavam desnutrição segundo avaliação da CB e CMB. A CP indicou depleção de massa muscular entre as mulheres, e a DCT obesidade apenas entre os homens. Encontrou-se consumo insuficiente de fibras, cálcio, vitaminas $D$ e $E$, enquanto gordura saturada, ferro e proteínas $(\mathrm{g} / \mathrm{kg})$ estavam acima da recomendação em ambos os sexos. Houve correlação fraca das variáveis antropométricas com a caloria total, macronutrientes e fibras. Assim, pôde-se confirmar o estado nutricional dos idosos institucionalizados, marcado por excesso de peso coexistente com perda muscular, e conclui-se que este esteja relacionado à distribuição dos macronutrientes, principalmente no que se refere ao percentual de lipídeos e carboidratos. Sugere-se que este quadro esteja agravado pelo sedentarismo dos idosos, uma vez que o consumo aumentado de proteínas não preveniu a perda de massa muscular.
\end{abstract}

Palavras-chave: Nutrição do idoso. Saúde do idoso institucionalizado. Consumo de alimentos.

NUTRITIONAL STATUS OF ELDERLY RESIDENTS IN A GERIATRIC INSTITUTION AND THE RELATION WITH FOOD CONSUMPTION

\section{ABSTRACT}

The presented work aimed to assess the nutritional status and food consumption of institutionalized elderly people, verifying possible relationships between them. A cross-sectional descriptive study with a quantitative approach was used, involving 48 elderly people. Weight (P), height (A), body mass index (BMI), arm circumferences (AC), calf (CC) and waist (WC), triceps skinfold (TSF), and arm muscle circumference were evaluated (AMC). The three-day $24 \mathrm{~h}$ food record (R24h) was used to analyze food consumption and was applied to the institution's caregivers. Spearman's correlation was utilized to verify the relationship between anthropometry and food consumption. From this survey, it was found that the majority of the elderly were overweight and experienced abdominal adiposity according to $\mathrm{BMI}$ and $\mathrm{WC}$, but presented malnutrition according to the assessment of $A C$ and AMC. CC indicated depletion of muscle mass among women, and TSF indicated obesity only among men. The consumption of fibers, calcium, vitamins $D$ and $E$ was insufficient, while saturated fat, iron and proteins $(\mathrm{g} / \mathrm{kg}$ ) were above the recommendation in both sexes. There was a weak correlation between anthropometric variables and total calories, macronutrients and fibers. Thus, it was possible to confirm the nutritional status of institutionalized elderly, characterized by excess weight coexisting with muscle loss, and it is concluded that this is related to the distribution of macronutrients, especially with regard to the percentage of lipids and carbohydrates. This condition is suggested to be impaired by the sedentary lifestyle of the elderly, since the increased protein consumption did not prevent the loss of muscle mass.

Keywords: Elderly nutrition. Health of the institutionalized elderly. Food consumption. 


\section{INTRODUÇÃO}

O envelhecimento é um processo dinâmico e progressivo, caracterizado por alterações fisiológicas, bioquímicas e morfológicas, mas também nutricionais. Com o envelhecimento observam-se mudanças na sensibilidade gustativa do idoso, havendo recusa pelos sabores azedo e amargo, e maior preferência pelos alimentos doces e salgados. Como consequência, o idoso apresenta prejuízos na qualidade da sua alimentação, caracterizada pela ingestão insuficiente de fibras e água, a qual contribui para a ocorrência de desidratação, constipação e doenças metabólicas, como o diabetes mellitus, a hipertensão arterial e as doenças cardiovasculares (SERGI et al., 2017).

Entre as modificações que ocorrem na composição corporal, observa-se perda da massa muscular associada à redução na força muscular e desempenho físico, mas também aumento do tecido adiposo. Além disso, achados mostram alta prevalência da obesidade sarcopênica na população idosa, a qual se caracteriza pela coexistência de desnutrição e adiposidade aumentada (BERNARDO; AMARAL, 2016). Assim, a avaliação nutricional do idoso é extremamente relevante, pois, mesmo com maior ocorrência de sobrepeso, os idosos ainda apresentam percentual elevado para risco de desnutrição (CARDOZO et al., 2017).

Sugere-se, portanto, uma relação entre o hábito alimentar dos idosos e a antropometria (SALGUEIRO et al., 2018). Estudo prévio mostrou que idosos institucionalizados apresentam maior prevalência de risco nutricional, principalmente quando aparece redução na mobilidade e capacidade cognitiva, e também entre aqueles que residem em instituições públicas (SILVÉRIO et al., 2016). Ainda, em instituições geriátricas onde as necessidades calóricas, proteicas e de micronutrientes não são atendidas, aponta-se maior risco de desnutrição e sarcopenia (REJÓN; LÓPEZ; ARTACHO, 2019). Diante do exposto, o objetivo deste estudo foi avaliar o estado nutricional e o consumo alimentar de idosos institucionalizados, verificando-se possíveis relações entre eles.

\section{METODOLOGIA}

\section{Delineamento do estudo}

Tratou-se de um estudo transversal descritivo, com abordagem quantitativa, a respeito do consumo alimentar e estado nutricional de 48 idosos residentes em uma instituição filantrópica localizada no Noroeste de Minas Gerais, na região do Alto Paranaíba, cujo município possuía aproximadamente 152.500 habi- tantes. A coleta dos dados ocorreu entre os meses de junho e julho de 2019. Como realidade social, os idosos raramente recebem visita familiar, e possuem auxílio de cuidadores para execução de suas atividades diárias. A instituição conta, também, com a prestação de serviço de uma nutricionista para a elaboração do cardápio, adequação da consistência das refeições e prescrição das dietas enterais.

Foram convidados a participar da pesquisa idosos de ambos os sexos com idade acima de 60 anos, residentes permanentes na instituição geriátrica. Foram incluídos todos aqueles que se disponibilizaram, não havendo exclusão em razão da presença de patologias prevalentes na terceira idade, como diabetes tipo 2, hipertensão arterial, osteoporose, doenças reumáticas e pulmonares, ou cognitivas, como demência, Alzheimer, entre outras. Foram incluídos os ambulantes, cadeirantes e acamados, idosos edêntulos (parcial e totalmente) e todos os que estavam em alimentação oral. Assim, foram excluídos eventuais residentes da instituição com idade inferior a 60 anos, ou os que se encontravam em nutrição enteral.

\section{Avaliação antropométrica}

Para a avaliação e classificação do estado nutricional dos idosos, foram coletadas as variáveis antropométricas de peso $(\mathrm{P})$ e altura $(\mathrm{A})$ para o cálculo do índice de massa corporal (IMC), circunferências do braço (CB), da panturrilha (CP) e da cintura (CC), dobra cutânea do tríceps (DCT) e circunferência muscular do braço (CMB). Na coleta dos dados, para os idosos capazes de deambular o peso corporal foi aferido por uma balança digital, posicionada em um local plano e firme. Já a altura foi aferida por fita métrica afixada em uma parede ausente de rodapé. Para aqueles incapazes de se manterem em posição ereta, o peso e a altura foram estimados pelas equações de Chumlea et al. (1988) e Chumlea, Guo e Steinbaugh (1994). O IMC $\left(\mathrm{kg} / \mathrm{m}^{2}\right)$ foi classificado conforme Lipschitz (1994).

A CP foi mensurada na perna esquerda, na sua parte mais protuberante, com o idoso com a perna dobrada, formando um angulo de 90 ㅇ graus com o joelho. Foi considerada adequada CP maior ou igual a 31 centímetros para homens e mulheres (WHO, 1995). A CC foi mensurada no ponto médio entre o rebordo costal inferior e a crista ilíaca anterossuperior. A CB foi mensurada no braço não dominante, com a fita métrica inelástica no ponto médio entre a extremidade da proeminência do olecrano e da ulna. A DCT foi mensurada tomando-se as mesmas regiões anatômicas, porém sobre o músculo do tríceps (LOHMAN; ROCHE; MARTORELL, 1988). A CMB foi obtida a partir da CB 
e da DCT (CMB = CB - DCT x 0,314). O percentual de adequação da CB, CMB e DCT foi classificado segundo Blackburn e Thornton (1979), e o ponto de corte da CC, considerado risco cardiometabólico, foi a medida maior ou igual a 94 centímetros para os homens e 80 centímetros para as mulheres (WHO, 1998).

\section{Avaliação do consumo alimentar}

Os dados de consumo alimentar foram obtidos por meio do recordatório de 24 horas (R24h) de três dias (dois dias semanais não consecutivos e um dia do fim de semana), com a finalidade de quantificar o consumo diário energético e de nutrientes (macro e micronutrientes) dos idosos. Para a aplicação do R24h buscou-se interagir com todos os idosos, entretanto, por se tratar de uma população com redução na memória e capacidade cognitiva, os dados relacionados ao consumo alimentar foram obtidos com os cuidadores da instituição, não havendo exclusão, portanto, de nenhum dos idosos em razão de doenças como demência ou Alzheimer. Estes dados foram coletados em medida caseira e forma de preparo dos alimentos e, em seguida, convertidos em peso (g) ou volume (ml). A ingestão foi estimada utilizando-se tabelas de composição de alimentos inseridas no software Diet Box. $A$ adequação do consumo alimentar foi verificada conforme as Dietary Reference Intakes (DRIs) do Institute of Medicine (PADOVANI et al., 2006) para esta faixa etária ( $\geq 60$ anos).

\section{Questões éticas}

O protocolo de pesquisa foi elaborado respeitando a Resolução no 466 de 2012 do Conselho Nacional de Saúde (CNS). O mesmo foi submetido e aprovado pelo Comitê de Ética e Pesquisa (CEP) do Centro Universitário de Patos de Minas - Unipam -, sob o parecer $n=3.380 .652$. Após a aprovação e previamente à abordagem dos idosos, o responsável pela instituição foi contatado para que este tomasse conhecimento do objetivo, justificativa e metodologia da pesquisa. Mediante a liberação por ele concedida, os pesquisadores estabeleceram contato com os idosos para iniciarem a coleta dos dados. Primeiramente, por se tratar de uma população em condição asilar, um Miniexame do Estado Mental (MEEM) foi aplicado aos idosos para verificação da capacidade cognitiva, e encontrou-se escore para demência (BVS, 2019). Em razão disso, o Termo de Consentimento Livre e Esclarecido (TCLE) foi assinado pelo responsável pela instituição e, consequentemente, pelos idosos, e os dados do consumo alimentar foram obtidos com os cuidadores da instituição.

\section{Análise estatística}

Para a realização das análises estatísticas utilizou-se o software IBM SPSS Statistics (v. 21, SPSS: IBM Company, Chicago, IL, USA). Os dados foram apresentados em prevalência ou média e desvio padrão. A normalidade das variáveis quantitativas contínuas foi verificada pelo teste Kolmogorov-Smirnov. O teste t para uma amostra foi utilizado para analisar a adequação do consumo alimentar segundo as DRIs (PADOVANI et al., 2006), e o teste de correlação bivariada de Spearman foi aplicado para verificar a relação das variáveis antropométricas com a ingestão calórica e de nutrientes. O nível de significância considerado foi menor ou igual a $5 \%(p \leq 0,05)$.

\section{RESULTADOS}

A referida instituição é financeiramente mantida e administrada por uma organização social local. Além disto, todos os residentes recebem aposentadoria no valor de um salário mínimo, a qual é utilizada para o custeio dos gastos do local e necessidades dos idosos. Na abordagem inicial contabilizou-se um total de 70 idosos. Destes, foram excluídos $7 \mathrm{com}$ idade inferior a 60 anos, 6 que se negaram a participar e 9 que estavam em nutrição enteral. Foram envolvidos no estudo, portanto, 48 idosos, sendo 16 homens e 32 mulheres.

A Tabela 1 apresenta as prevalências das variáveis investigadas. Encontrou-se que a maioria dos idosos não frequentou a escola, era capaz de deambular e apresentava dentição parcial. Entre os homens, a maioria estava na faixa etária de 70 a 79 anos $(43,8 \%)$ e tinha funcionamento intestinal irregular (62,5\%). Já as mulheres estavam, em sua maioria, com idade superior a 80 anos $(43,8 \%)$ e a maior parte apresentava hábito intestinal regular $(68,8 \%)$.

$\mathrm{Na}$ avaliação do estado nutricional, observou-se que a maioria dos idosos, de ambos os sexos, estava com excesso de peso segundo a classificação do IMC, e com risco para complicações cardiometabólicas, conforme a medida da CC. A adequação da $\mathrm{CB}$ e da $\mathrm{CMB}$, no entanto, indicou maior prevalência de desnutrição. Já a adequação da DCT apontou excesso de peso entre os homens e desnutrição entre as mulheres, e a partir da avaliação da CP apenas as mulheres apresentavam também depleção de massa muscular. 
Tabela 1 - Perfil epidemiológico e nutricional de idosos residentes em uma instituição geriátrica no interior de Minas Gerais

\begin{tabular}{|c|c|c|}
\hline \multirow[b]{3}{*}{ Idade } & \multicolumn{2}{|c|}{ Prevalência \% (n) } \\
\hline & $\begin{array}{c}\text { Homem } \\
(n=16)\end{array}$ & $\begin{array}{c}\text { Mulher } \\
(n=32)\end{array}$ \\
\hline & \\
\hline 60 a 69 anos & $31,2(05)$ & $21,8(07)$ \\
\hline 70 a 79 anos & $43,8(07)$ & $34,4(11)$ \\
\hline 80 anos ou mais & $25,0(04)$ & $43,8(14)$ \\
\hline \multicolumn{3}{|l|}{ Escolaridade } \\
\hline Não frequentou a escola & $87,5(14)$ & $87,5(28)$ \\
\hline Fundamental incompleto & $12,5(02)$ & $12,5(04)$ \\
\hline \multicolumn{3}{|l|}{ Capacidade de deambular } \\
\hline Sim & $56,2(09)$ & $62,5(20)$ \\
\hline Não & $43,8(07)$ & $37,5(12)$ \\
\hline \multicolumn{3}{|l|}{ Funcionamento intestinal } \\
\hline Regular & $37,5(06)$ & $68,8(22)$ \\
\hline Irregular & $62,5(10)$ & $31,2(10)$ \\
\hline \multicolumn{3}{|l|}{ Dentição } \\
\hline Presença parcial & $50,0(08)$ & $59,4(19)$ \\
\hline Prótese dentária & $25,0(04)$ & $21,9(07)$ \\
\hline Edêntulo & $25,0(04)$ & $18.7(06)$ \\
\hline \multicolumn{3}{|l|}{ Estado nutricional ${ }^{\#}$} \\
\hline Baixo peso & $18,8(03)$ & $18,7(06)$ \\
\hline Eutrofia & $25,0(04)$ & $34,4(11)$ \\
\hline Excesso de peso & $56,2(09)$ & $46,9(15)$ \\
\hline \multicolumn{3}{|l|}{ Classificação da CC } \\
\hline Com risco nutricional & $56,2(09)$ & $68,8(22)$ \\
\hline Sem risco nutricional & $43,8(07)$ & $31,2(10)$ \\
\hline \multicolumn{3}{|l|}{ Classificação da CP } \\
\hline Com depleção & $31,2(05)$ & $50,0(16)$ \\
\hline Sem depleção & $68,8(11)$ & $50,0(16)$ \\
\hline \multicolumn{3}{|l|}{ Classificação da CB } \\
\hline Desnutrição & $68,8(11)$ & $43,8(14)$ \\
\hline Eutrofia & $31,2(05)$ & $37,5(12)$ \\
\hline Excesso de peso & $00,0(00)$ & $18,7(06)$ \\
\hline \multicolumn{3}{|l|}{ Classificação da CMB } \\
\hline Desnutrição & $87,5(14)$ & $40,6(13)$ \\
\hline Eutrofia & $12,5(02)$ & $34,4(11)$ \\
\hline Excesso de peso & $00,0(00)$ & $25,0(08)$ \\
\hline \multicolumn{3}{|l|}{ Classificação da DCT } \\
\hline Desnutrição & $00,0(00)$ & $62,5(20)$ \\
\hline Eutrofia & $00,0(00)$ & 34,4 (11) \\
\hline Excesso de peso & $100,0(16)$ & $03,1(01)$ \\
\hline \multirow{2}{*}{$\begin{array}{l}\text { Variáveis antropométricas } \\
\text { Idade (anos) }\end{array}$} & Média $\pm D P$ & Média $\pm D P$ \\
\hline & $74,9 \pm 9,9$ & $78,8 \pm 10,5$ \\
\hline $\mathrm{IMC}\left(\mathrm{kg} / \mathrm{m}^{2}\right)$ & $28,6 \pm 6,6$ & $28,0 \pm 7,5$ \\
\hline $\mathrm{CC}(\mathrm{cm})$ & $94,8 \pm 14,1$ & $89,1 \pm 15,9$ \\
\hline $\mathrm{CP}(\mathrm{cm})$ & $32,2 \pm 3,5$ & $30,3 \pm 6,2$ \\
\hline $\mathrm{CB}(\mathrm{cm})$ & $26,6 \pm 3,6$ & $27,3 \pm 6,0$ \\
\hline $\mathrm{CMB}(\mathrm{cm})$ & $20,0 \pm 3,9$ & $21,9 \pm 5,9$ \\
\hline $\mathrm{DCT}(\mathrm{mm})$ & $20,9 \pm 4,9$ & $17,1 \pm 2,2$ \\
\hline
\end{tabular}

CC: circunferência da cintura. CP: circunferência da panturrilha. CB: circunferência do braço. CMB: circunferência muscular do braço. DCT: dobra cutânea do tríceps (LOHMAN; ROCHE; MARTORELL, 1988). DP: desvio padrão. IMC: índice de massa corporal. \#O estado nutricional foi classificado pelo IMC (kg/m2), segundo Lipschitz (1994), a CC e CP conforme a WHO (1998; 1995, respectivamente), e adequação da CB, CMB e DCT de acordo com Blackburn e Thornton (1979).

Fonte: Os autores, 2019.
Utilizando o recordatório de 24 horas de três dias, foram obtidos resultados quantitativos referentes ao consumo energético e de macro e micronutrientes pelos idosos, os quais estão apresentados na Tabela 2. Uma vez que a aplicação deste instrumento ocorreu mediante entrevista com os cuidadores da instituição, e em razão do fato de os homens e as muIheres ficarem em ambientes separados, a coleta dos dados alimentares de cada sexo foi realizada em dias distintos, havendo, portanto, diferença na composição do cardápio fornecido. Considerou-se, entretanto, que este não foi um fator limitante para o estudo, pois a análise de adequação, segundo as DRIs, considera o sexo do indivíduo.

Encontrou-se que as mulheres tinham consumo calórico $11,5 \%$ acima do recomendado pela Estimated Energy Requirement (ERR), enquanto os homens apresentavam apenas $78 \%$ de adequação do consumo de energia necessário. Na avaliação dos macronutrientes, os idosos de ambos os sexos apresentavam percentual de consumo dentro da Acceptable Macronutrient Distribution Range (AMDR), estando as muIheres com consumo de carboidratos mais próximo ao limite superior (56\%) e os homens no limite inferior $(45,2 \%)$. As proteínas foram avaliadas de acordo com a Dietary Reference Intake (RDA), estando o consumo por ambos os sexos acima do valor mínimo recomendado (1,1 g PTN $/ \mathrm{kg}$ entre os homens e $1,2 \mathrm{~g}$ PTN $/ \mathrm{kg}$ entre as mulheres). A recomendação dos carboidratos e lipídeos em gramas foi determinada a partir da AMDR ( $45 \%$ a $65 \%$ e $20 \%$ a $35 \%$, respectivamente). Para tanto, a recomendação das calorias totais foi previamente estabelecida, levando-se em consideração as variáveis componentes da EER, ou seja, sexo, idade (em anos), altura (em metros) e peso (em quilogramas), e o fator atividade física igual a 1 (Tabela 2).

No que se refere aos micronutrientes, segundo a RDA observou-se consumo insuficiente de fibras, gordura monoinsaturada, cálcio e das vitaminas $\mathrm{D}$ e $\mathrm{E}$ por ambos os sexos, enquanto o consumo de ferro, selênio e gorduras saturadas mostrou-se acima do limite de recomendação. O percentual de açúcar (equivalente ao açúcar de adição em alguns produtos processados, como os cereais servidos na forma de mingau) não ultrapassou significativamente o limite máximo diário aceitável (10\%). O consumo em gramas, entretanto, foi superior $(p<0,01)$ aos $25 \mathrm{~g} / \mathrm{dia}$ recomendado pela OMS (42g entre os homens e $43 \mathrm{~g}$ entre as mulheres). Discrepância entre os sexos foi observada para o consumo de vitaminas A, C e B12, gordura poli-insaturada, trans e colesterol, além do sódio. 
Tabela 2 - Ingestão alimentar diária de macro e micronutrientes de idosos residentes em uma instituição geriátrica no interior de Minas Gerais

\begin{tabular}{|c|c|c|c|c|c|c|}
\hline $\begin{array}{l}\text { Nutrientes } \\
\text { (dia) }\end{array}$ & Homens & $\begin{array}{l}\text { Recomendação } \\
\text { (DRI) }\end{array}$ & $\begin{array}{c}\% \\
\text { Adequação }\end{array}$ & Mulheres & $\begin{array}{l}\text { Recomendação } \\
\text { (DRI) }\end{array}$ & $\begin{array}{c}\% \\
\text { Adequação }\end{array}$ \\
\hline Fibra (g) & $9,8 \pm 2,8^{\bar{\top}}$ & 30 & 32,7 & $16,9 \pm 6,0^{\bar{\top}}$ & 21 & 80,5 \\
\hline Açúcar (\%)* & $11,0 \pm 5,5$ & $<10$ & 110 & $10,2 \pm 4,0$ & $<10$ & 102 \\
\hline G. Saturada (\%)* & $18,9 \pm 11,6^{\top}$ & $<10$ & 189 & $15,7 \pm 4,8^{\bar{\top}}$ & $<10$ & 157 \\
\hline G. Poli-ins (\%)*\# & $17,9 \pm 12,8^{\bar{\top}}$ & $6-10$ & 223.7 & $7,0 \pm 2,1^{\bar{\top}}$ & $6-10$ & 87,5 \\
\hline G. Monoins(\%)* & $12,1 \pm 6,2^{\bar{\top}}$ & $<20$ & 60,5 & $8,1 \pm 3,2^{\bar{\top}}$ & $<20$ & 40,5 \\
\hline G. Trans (\%) & $1,4 \pm 2,2$ & $<1$ & 140 & $0,3 \pm 0,2^{\bar{\top}}$ & $<1$ & 30,0 \\
\hline Colesterol (mg) & $288 \pm 107$ & 300 & 96,1 & $270 \pm 48^{\bar{\top}}$ & 300 & 89,9 \\
\hline Sódio (mg) & $1079 \pm 193^{\bar{\top}}$ & 1200 & 89.9 & $2098 \pm 597^{\bar{\top}}$ & 1200 & 174,9 \\
\hline Ferro (mg) & $17,3 \pm 13,9^{\bar{\top}}$ & 8 & 216,2 & $13,8 \pm 2,7^{\bar{\top}}$ & 8 & 172,5 \\
\hline Zinco (mg) & $12,3 \pm 6,0$ & 11 & 111,8 & $7,8 \pm 2,1$ & 8 & 97,5 \\
\hline Selênio ( $\mu \mathrm{g})$ & $131 \pm 41^{\bar{\top}}$ & 55 & 239,1 & $70,3 \pm 27,8^{\bar{\top}}$ & 55 & 127,8 \\
\hline Cálcio (mg) & $504 \pm 211^{\bar{\top}}$ & 1200 & 42,0 & $765 \pm 210^{\bar{\top}}$ & 1200 & 63,7 \\
\hline Vit. D ( $\mu \mathrm{g})$ & $5,0 \pm 3,6^{\bar{\top}}$ & 15 & 33,3 & $2,1 \pm 0,5^{\bar{\top}}$ & 15 & 14,0 \\
\hline Vit. E (mg) & $3,8 \pm 2,2^{\bar{\top}}$ & 15 & 25,3 & $8,0 \pm 3,0^{\bar{\top}}$ & 15 & 53,3 \\
\hline Vit. C (mg) & $39,2 \pm 16,8^{\bar{\top}}$ & 90 & 43,5 & $107 \pm 37^{\bar{\top}}$ & 75 & 142,7 \\
\hline Vit. A ( $\mu \mathrm{g})$ & $803 \pm 194$ & 900 & 89,2 & $1773 \pm 557^{\bar{\top}}$ & 700 & 253,2 \\
\hline Vit. B6 (mg) & $1,7 \pm 1,0$ & 1,7 & 100 & $1,5 \pm 0,4$ & 1,5 & 100 \\
\hline Vit. B12 ( $\mu \mathrm{g})$ & $5,8 \pm 2,6^{\bar{\top}}$ & 2,4 & 241,7 & $2,6 \pm 0,6$ & 2,4 & 108,3 \\
\hline $\mathrm{CHO}(\mathrm{g})^{*}$ & $166,1 \pm 38$ & 212 a 306 & 45,2 & $237,2 \pm 39$ & 173 a 250 & 56,0 \\
\hline $\operatorname{LIP}(\mathrm{g})^{*}$ & $46,9 \pm 9,5$ & 42 a 73 & 30,1 & $54,6 \pm 11,4$ & 34 a 60 & 28,8 \\
\hline PTN $(g)^{*}$ & $76,0 \pm 17$ & 47 a 164 & 21,1 & $76,5 \pm 14$ & 38 a 135 & 18,0 \\
\hline Calorias (kcal) & $1465 \pm 281^{\bar{\top}}$ & $1880 \pm 351$ & 78,2 & $1714 \pm 340^{\bar{\top}}$ & $1537 \pm 255$ & 111,5 \\
\hline
\end{tabular}

Homens, $n=16$. Mulheres, $n=32$. Os valores dos nutrientes representam a média do consumo alimentar diário dos três dias de coleta do R24h. Os valores de recomendação para calorias e macronutrientes foram determinados a partir da EER de cada idoso, e estão apresentados como média, de acordo com o sexo. ${ }^{\top} H o u v e$ diferença estatística $(p \leq 0,05)$ das DRIs pelo teste t para uma amostra (SPSS). *O percentual foi calculado segundo o VET (valor energético total) diário ingerido. "O percentual de adequação foi calculado segundo o valor médio de recomendação para as gorduras poli-insaturadas, ou seja, 8\% (SBC, 2017). CHO: carboidrato. LIP: lipídeo. PTN: proteína.

Fonte: Os autores, 2019.

Com a finalidade de verificar se estas inadequações nutricionais estariam relacionadas com a avaliação antropométrica, a correlação de Spearman foi conduzida tomando-se as variáveis de IMC, CC, CP, CB, $\mathrm{DCT}$, calorias, carboidratos, proteínas, lipídeos, gorduras saturada, fibra, vitamina $D$, cálcio, açúcar e sódio. Para tal análise, os idosos foram considerados grupo único. Houve correlação do IMC apenas com as proteínas $(\rho=0,293 ; p=0,043)$. A CP esteve correlacionada com as proteínas $(\rho=0,355 ; p=0,013)$ e vitamina $D$ $(\rho=0,438 ; p=0,002)$. A DCT apresentou correlação negativa com os carboidratos $(\rho=-0,291 ; p=0,045)$. A CC não mostrou correlação com os nutrientes.

Já a CB esteve correlacionada com as proteínas $(\rho=0,321 ; p=0,026)$, carboidratos $(\rho=0,415$; $p=0,003)$, calorias $(\rho=0,379 ; p=0,008)$, gordura saturada $(\rho=0,342 ; p=0,017)$ e com as fibras $(\rho=0,295$; $\mathrm{p}=0,042)$. Do mesmo modo, a CMB apresentou correlação com as proteínas $(\rho=0,326 ; p=0,024)$, carboidratos $(\rho=0,490 ; p<0,001)$, calorias $(\rho=0,433 ; p=0,002)$, gordura saturada $(\rho=0,308 ; p=0,033)$ e fibras $(\rho=0,342$; $p=0,017)$.

\section{DISCUSSÃO}

O presente estudo propôs-se a avaliar o estado nutricional e o consumo alimentar de idosos institucionalizados no interior de Minas Gerais e a possível relação entre estes fatores. No que se refere ao objetivo do trabalho, encontrou-se prevalência de idosos com sobrepeso, circunferência da cintura aumentada e desnutrição segundo a adequação da circunferência do braço e da circunferência muscular do braço. Além disso, o consumo de fibras, gordura monoinsaturada, 
cálcio e das vitaminas D e E mostrou-se insuficiente, enquanto o consumo de ferro, gordura saturada e proteínas (g PTN/kg) estava acima do recomendado.

A predominância de mulheres reflete os dados do último levantamento do IBGE (2018), o qual mostrou que $56 \%$ da população idosa brasileira corresponde ao sexo feminino. Além disso, como aqui observado, outra característica do idoso brasileiro é a baixa escolaridade, associada a uma menor renda econômica e menor acesso aos serviços públicos, os quais podem comprometer a qualidade de vida destes indivíduos (SANTOS et al., 2018).

Várias alterações fisiológicas são observadas no organismo do idoso, as quais trazem prejuízos à mobilidade, à cognição e ao estado clínico e nutricional. Embora a maioria dos idosos fosse capaz de deambular, eles não possuíam independência para gerenciamento da própria vida, como tomar banho, trocar de roupa, ir ao banheiro, cozinhar e se alimentar, fatores apontados como os principais para a condição de asilo de muitos idosos, juntamente com o comprometimento cognitivo (LINI; PORTELLA; DORING, 2016).

Esta dificuldade observada nos idosos para realizar as atividades diárias indica perda de sua funcionalidade, e pode estar associada às mudanças na composição corporal, sendo observados redução da massa magra e aumento do tecido adiposo (BERNARDO; AMARAL, 2016). Foi verificado, anteriormente, que idosas com excesso de peso pelo IMC apresentaram habilidade reduzida para a mobilidade física, menor capacidade de força nos membros superiores e prejuízo na resistência aeróbia (VAGETTI et al., 2017). Assim como encontrado no presente estudo, tem-se observado sobreposição do número de idosos com IMC de excesso de peso ante os eutróficos e os desnutridos. Além do IMC, a CC aumentada e a CP dentro da normalidade são outra realidade do idoso contemporâneo (CARDOZO et al., 2017; SALGUEIRO et al., 2018; PEREIRA; SAMPAIO, 2019). Mais da metade dos idosos avaliados, de ambos os sexos, apresentava medida da CC acima do ponto de corte considerado adequado. $\mathrm{O}$ aumento na CC traz preocupação, pois indica acúmulo de tecido adiposo abdominal e está relacionado ao maior risco para as doenças cardiometabólicas, como hipertensão arterial, diabetes, dislipidemias e aterosclerose (SBC, 2017).

No presente estudo as patologias dos idosos não foram investigadas, porém, mediante relatos dos cuidadores da instituição, estas eram presentes. Os pontos de corte para risco cardiometabólico utilizados para esta faixa etária são os mesmos usados para os adultos (WHO, 1998). Estudo recente, entretanto, se propôs a estabelecer um marcador antropométrico de excesso de peso em idosos brasileiros a partir da razão cintura-estatura, cujo ponto de corte seria 0,55 (CORRÊA et al., 2017). Ainda, quando tal relação foi aplicada em adultos, esta mostrou-se mais associada aos fatores de risco cardiovascular do que a CC isolada (BARROSO et al., 2017). Assim, baseado nesta razão, entre os idosos aqui avaliados $81 \%$ dos homens e $62,5 \%$ das mulheres estariam com excesso de peso.

A DCT indica a reserva de gordura subcutânea, e sua análise tem sido sugerida como parâmetro para avaliação das reservas de gordura corporal no idoso. Segundo sua classificação, tem-se observado alta prevalência de tecido adiposo em excesso nesta população, assim como a CC aumentada (OLIVEIRA et al., 2019). Entre os idosos avaliados, apenas os homens apresentaram obesidade pela medida da DCT, enquanto as mulheres mostraram desnutrição. Achados semelhantes foram observados anteriormente, e, após adequação da DCT, encontrou-se maior prevalência de desnutrição em idosas institucionalizadas, estando a maior parte dos homens sob a classificação de excesso de peso (SCHMIDT et al., 2017).

No presente estudo, apesar de grande parte dos idosos apresentarem IMC (50\%) e CC (67\%) acima do ideal de 94 centímetros para os homens e 80 centímetros para as mulheres (WHO, 1998), e nos homens (100\%) ainda a DCT acima do percentil 90 (FRISANCHO, 1981, 1990), não se pode ignorar que para $31 \%$ dos homens e $50 \%$ das mulheres a medida da $\mathrm{CP}$ indicou depleção de massa muscular. Este achado causa preocupação, pois idosos com CP inferior a 31 centímetros possuem pior prognóstico clínico, principalmente quando hospitalizados, por estarem mais sujeitos ao óbito (MELLO; WAISBERG; SILVA, 2016). Além disso, a avaliação da CP também é recomendada na atenção primária ao idoso. Um estudo observou que entre os idosos, CP inferior a 34 centímetros em homens e 33 centímetros em mulheres já indicavam massa muscular diminuída (PAGOTTO et al., 2018), limiares estes acima do observado nos idosos aqui avaliados ( $32 \pm 3$ centímetros e $30 \pm 6$ centímetros respectivamente).

A redução da massa magra na região dos membros é relatada na avaliação nutricional do idoso, mesmo quando observado o excesso de peso e de gordura abdominal (OLIVEIRA et al., 2019). No presente estudo, a CB e a CMB mostraram-se inferiores ao percentil 50 para ambos os sexos, e o percentual de adequação destas duas variáveis indicou processo de desnutrição (FRISANCHO, 1981, 1990). Achados prévios acerca do estado nutricional de idosos institucionalizados mos- 
traram desnutrição leve e moderada segundo a medida da $\mathrm{CB}$, porém, mediante a avaliação da $\mathrm{CMB}$, a desnutrição foi encontrada apenas entre os homens, estando as mulheres em classificação de eutrofia (SCHMIDT et al., 2017).

Tal discrepância entre os sexos e os diferentes estudos pode ser explicada pelo fato de que o declínio do estado nutricional ocorre conforme a progressão das doenças de base do idoso, principalmente no que se refere às doenças neurodegenerativas (GOULART et al., 2017). Neste contexto, idosos institucionalizados e com declínio cognitivo têm maior risco nutricional (DAMO et al., 2018). Ainda, idosos em condição de fragilidade foram aqueles com idade mais avançada, em sua maioria mulheres, e que apresentavam maior perda de massa muscular concomitante à maior adiposidade central (MELLO et al., 2017), perfil este, o da amostra avaliada no presente estudo, que apresentava, também, quadro de demência.

A condição de fragilidade no idoso também interfere no consumo alimentar. Este apresenta maior uso de cereais e redução no consumo de frutas e leguminosas (feijão). Independente do grau de fragilidade, entretanto, idosos apresentam baixo consumo de produtos lácteos e tendência para menor consumo de vegetais, enquanto o consumo de carnes e produtos ricos em açúcares e gorduras se mostra alto (MELLO et al., 2017). Estes hábitos podem ser decorrentes das mudanças na sensibilidade gustativa do idoso, o qual tende a recusar os sabores azedo e amargo e opta pelos alimentos doces e salgados (SERGI et al., 2017).

Como a instituição geriátrica contava com o serviço de uma nutricionista para a elaboração do cardápio na aplicação do R24h, percebeu-se que não havia oferta de produtos ultraprocessados, salvo em datas comemorativas. Além disto, atentava-se em controlar a adição de sal e açúcar às preparações, porém, por se tratar de uma instituição filantrópica, o uso de alimentos reduzidos nestes nutrientes não podia ser sempre garantido, uma vez que recebiam doação da comunidade local. Assim, os idosos consumiam, rotineiramente, arroz e feijão, verduras e tubérculos cozidos e em molho. $O$ consumo de frutas ocorria, comumente, em vitaminas preparadas com leite, e a fonte proteica mais comum era a carne de frango cozida, seguida da carne bovina moída e ovos. Uma preparação ofertada com frequência eram os mingaus à base de cereais (aveia, maisena, trigo) e leite.

Mesmo, entretanto, havendo presença dos diferentes grupos alimentares na dieta dos idosos, encontraram-se inadequações no que se refere à necessidade diária de fibras, gorduras monoinsaturadas, cálcio e as vitamina $\mathrm{D}$ e $\mathrm{E}$, assim como o excesso de ferro, proteínas e gordura saturada. Entre as causas para inadequação alimentar por idosos institucionalizados, destacou-se o recente tempo de internação e o número elevado de medicamentos, mas, principalmente, o sentimento de tristeza e a falta de apetite (SILVA et al., 2019a).

A baixa ingestão de fibras favorece a constipação intestinal, e os idosos têm maior tendência a esta em razão das alterações fisiológicas da idade, juntamente com a baixa ingestão de fibras e água (SERGI et al., 2017). Estudo prévio realizado com idosos institucionalizados encontrou baixa ingestão de fibras apenas entre os homens, porém a maior prevalência de constipação foi observada nas mulheres (SILVA et al., 2016). Diferentemente do observado no presente estudo, no qual os homens apresentaram percentual de inadequação para o consumo de fibras maior que as mulheres, mas eram os que possuíam, consequentemente, maior irregularidade no funcionamento intestinal.

Nesta instituição geriátrica, porém, o baixo consumo de fibras pode ser justificado pela presença frequente no cardápio de alimentos servidos em textura pastosa, cujo método de cocção reduz o teor de fibras, além das calorias e micronutrientes (REJÓN; LÓPEZ; ARTACHO, 2019). Esta prática ocorria a fim de facilitar a deglutição pelos idosos edêntulos, ou com dentição parcial, e ainda por aqueles com déficit cognitivo, os quais podem apresentar disfagia. Já foi verificado que a ausência da dentição ou o uso de próteses esteve associado à desnutrição em idosos institucionalizados (SAARELA et al., 2016). Utilizando-se a miniavaliação nutricional, encontrou-se associação entre a disfagia e o risco de desnutrição (SILVA et al., 2019b).

A deficiência de vitamina $D$ e cálcio é outra realidade no hábito alimentar do idoso, e causa preocupação para além do maior risco de osteoporose. Discussões recentes mostram que o desequilíbrio nestes dois micronutrientes contribui para o desenvolvimento da síndrome metabólica, caracterizada por um grupamento de fatores de risco para o diabetes e as doenças cardiovasculares, tais como aumento da glicose e dos lipídeos séricos, e da adiposidade central. Quando adequada, a vitamina D contribui para o controle da glicemia por meio da diminuição da resistência à insulina, e também contribui para a homeostase pressórica via regulação do sistema renina-angiotensina. Já o cálcio influencia o balanço sérico de lipídeos (absorção e excreção) e, portanto, o balanço de energia (PANNU et al., 2016). 
Uma vez que a vitamina $E$ tem papel antioxidante no organismo, por ser capaz de proteger as membranas celulares da oxidação durante os processos patológicos, a deficiência de vitamina E encontrada nos idosos do presente estudo também chamou a atenção. A literatura é consistente ao relacionar vias inflamatórias e oxidativas no desenvolvimento de doenças metabólicas, as quais são frequentes no idoso. Por exemplo, indivíduos pré-diabéticos, os quais apresentavam IMC e CC elevados, já apresentavam baixos níveis de vitamina $E$ e quadro de estresse oxidativo (RAMÍREZ et al., 2017).

Outro fator predisponente para o desenvolvimento de doenças metabólicas é o consumo insuficiente das gorduras insaturadas, concomitante ao consumo elevado de gordura saturada (SBC, 2017). No presente estudo este padrão alimentar foi observado para ambos os sexos, havendo ambiguidade apenas no consumo das gorduras poli-insaturadas. $O$ fornecimento insuficiente de gorduras poli-insaturadas foi encontrado no cardápio de outras instituições geriátricas, juntamente com alguns micronutrientes, sendo tais inadequações sugeridas como a causa de má nutrição em idosos institucionalizados (REJÓN; LÓPEZ; ARTACHO, 2019).

No que se refere às proteínas, os idosos tinham consumo dentro da AMDR ( $10 \%$ a $35 \%$ ) e acima da RDA. De fato, um levantamento a respeito do consumo alimentar de idosos brasileiros encontrou ingestão diária de $75,5 \mathrm{~g}$ de proteínas, sendo este o macronutriente com melhor adequação segundo a AMDR (PREVIDELLI; GOULART; AQUINO, 2017). Este achado é bem visto, uma vez que o consumo insuficiente de proteínas é relatado em estudos com idosos institucionalizados (SAARELA et al., 2016) e apontado como causa para a sarcopenia e a fragilidade destes (REJÓN; LÓPEZ; ARTACHO, 2019).

O consumo adequado de proteína, observado neste estudo, pode ser atribuído ao fato de os idosos receberem ao longo das seis refeições diárias ofertadas na instituição (desjejum, colação, almoço, lanche, jantar e ceia), preparações contendo leite e derivados, carnes e leguminosas, conforme encontrado no R24h. Como mostrado em estudo anterior, a distribuição equilibrada das fontes proteicas ao longo das refeições é uma boa estratégia para aumentar a ingestão de proteína em idosos (TIELAND et al., 2017).

Ainda em relação ao consumo frequente de carnes e leguminosas, salientam-se estes como pontos positivos no cardápio da instituição avaliada, pois eles também contribuíram para o consumo de ferro acima do mínimo recomendado, mas sem extrapolar a
UL (Tolerable Upper Intake Level). A ingestão adequada de ferro previne a anemia no idoso, principalmente quando o mineral está inserido numa dieta adequada em calorias, proteínas, gorduras e micronutrientes antioxidantes (BIANCHI, 2015).

Em relação aos demais macronutrientes, os carboidratos e os lipídeos também estiveram dentro da AMDR em ambos os sexos. Infere-se, entretanto, que o consumo de energia $11 \%$ acima do recomendado pelas mulheres e $22 \%$ abaixo do ideal pelos homens possa ser um reflexo do consumo dos carboidratos, os quais estiveram mais próximos ao limite máximo entre as mulheres e no limite inferior entre os homens (PADOVANI et al., 2006). Tais achados mostraram-se diferentes do observado na população brasileira idosa, na qual se encontrou ingestão similar de carboidratos (53\%) entre os sexos, mas maior ingestão calórica entre os homens (PREVIDELLI; GOULART; AQUINO, 2017).

De fato, estudos acerca do estado nutricional e consumo alimentar em idosos mostram que os homens possuem maior ingestão calórica, entretanto, ao observar o percentual energético dos macronutrientes, o presente estuda corrobora achados prévios, nos quais encontrou-se ingestão de carboidratos maior entre as mulheres e ingestão de proteínas e lipídeos ligeiramente superior entre os homens (SALGUEIRO et al., 2018). Ressalta-se, todavia, que, embora houvesse maior consumo energético e de carboidratos entre as mulheres, o excesso de peso, CC aumentada e depleção de massa muscular nos membros foi observada em ambos os sexos, havendo discrepância apenas na classificação da DCT.

No presente estudo, embora a CC tenha sido elevada na amostra, ela não se mostrou correlacionada com nenhum dos nutrientes. Verificou-se, contudo, correlação positiva entre o consumo de proteínas e as demais variáveis antropométricas avaliadas, com exceção da DCT. Já os carboidratos correlacionaram-se negativamente com a DCT, mas positivamente com a $\mathrm{CB}$ e a $\mathrm{CMB}$, assim como as calorias, gordura saturada e fibras, as quais se mostraram correlacionadas positivamente com estes marcadores braquiais de reserva tecidual. Por fim, além das proteínas, a vitamina $D$ também apresentou correlação positiva com a $\mathrm{CP}$, marcador de reserva muscular no membro inferior. Todas as correlações, porém, foram fracas $(\leq 0,40)$.

Diante destes achados e os apontados na literatura (PREVIDELLI; GOULART; AQUINO, 2017; MARTINS et al., 2016; SALGUEIRO et al., 2018), o consumo de macronutrientes, o qual reflete no consumo calórico, 
mostra-se como o fator determinante para o estado nutricional do idoso. Endossa-se esta proposição, pois, mesmo idosos que tinham consumo elevado de frutas, mas baixo consumo de carboidratos integrais, apresentaram associação com a obesidade, estando esta última relacionada também com doenças osteomusculares e cardiometabólicas (SILVEIRA et al., 2016). Ainda, a ingestão de lipídeo total mais próxima do limite superior (35\%) é outra característica da alimentação de idosos com excesso de peso e gordura corporal, e, embora o consumo de proteínas estivesse adequado e pudesse assegurar sua função anabólica, não se pode ignorar que a fonte proteica da dieta dos idosos também era fonte de gordura saturada (SALGUEIRO et al., 2018).

Chama a atenção, também, o fato de que a proteína por si só não foi suficiente para garantir IMC de eutrofia ou prevenir a depleção de massa muscular nos idosos avaliados. Sugere-se que a ausência de prática de atividade física na instituição geriátrica pode estar contribuindo para o quadro nutricional dos idosos. Estudo duplo cego randomizado encontrou que idosos obesos sob controle do consumo calórico, suplementados com proteína e vitamina $D$, e que executaram exercícios de força durante 13 semanas, tiveram preservação da massa muscular dos membros (VERREIJEN et al., 2015).

Por fim, aponta-se como limitação do estudo o número pequeno da amostra, fator que dificulta o estabelecimento de relações consistentes no que se refere à influência do consumo alimentar do idoso sobre o estado nutricional. O uso do R24h pode ser um fator limitante, uma vez que ele informa o consumo de alguns dias, mas não reflete o hábito alimentar. Ainda referente ao R24h, por se tratar de instrumento dependente da memória do indivíduo, a informação pode não ser fidedigna. Embora a coleta dos dados alimentares tenha ocorrido com os cuidadores da instituição, não se pode negar que havia um número limitado de profissionais para assistir todos os idosos do local, os quais dependem de auxílio inclusive para se alimentar. $O$ fato de a coleta destes dados ter ocorrido em dias diferentes para os homens e as mulheres, e terem sido encontradas algumas discrepâncias na ingestão alimentar, não é apontado como um fator limitante, pois as coletas foram realizadas por nutricionista. Além do mais, estudo com idosos em comunidade também encontrou diferença na ingestão de macro e micronutrientes entre os sexos (PEREIRA et al., 2018).
Sugere-se, entretanto, que outros estudos desta vertente sejam realizados, englobando um número amostral maior, e que, além dos modelos transversais, estudos de coorte sejam conduzidos, a fim de melhor estabelecer a relação entre o estado nutricional do idoso e seu hábito alimentar.

\section{CONCLUSÃO}

Diante deste estudo, conclui-se que, mesmo se tratando de indivíduos em situação asilar, o perfil nutricional dos idosos endossa o observado para esta faixa etária, ou seja, excesso de peso, acompanhado de adiposidade central e depleção de massa muscular tanto em membros inferiores quanto superiores. $\mathrm{O}$ consumo de micronutrientes também confirmou o observado para estes indivíduos, havendo deficiência de fibras, cálcio e vitamina D, juntamente com o consumo excessivo de gorduras saturadas. Sustenta-se, portanto, a hipótese de que o estado nutricional destes idosos esteja relacionado à distribuição dos macronutrientes no cardápio, situação agravada pela completa inatividade física dos mesmos, uma vez que o consumo adequado de proteínas não foi capaz de prevenir a depleção muscular.

Dentro do cenário atual que se observa para as instituições geriátricas filantrópicas, sugere-se maior cuidado na elaboração dos cardápios no que se refere à distribuição dos macronutrientes e no fornecimento dos micronutrientes, buscando-se técnicas de cocção que não reduzam bruscamente a densidade nutricional do alimento. Além disto, que se pense na adoção de programas de atividade física para estes idosos, visando a amenizar as mudanças fisiológicas da idade e melhorar o bem-estar emocional dos mesmos.

\section{AGRADECIMENTOS}

Os autores agradecem ao responsável pela instituição geriátrica filantrópica e também aos funcionários (equipe de nutrição e cuidadores dos idosos) por autorizarem a realização da pesquisa, bem como por a viabilizarem, fornecendo as informações dietéticas dos idosos.

\section{REFERÊNCIAS}

BARROSO, T. A. et al. Associação entre a obesidade central e a incidência de doenças e fatores de risco cardiovascular. International Journal of Cardiovascular Sciences, v. 30, n. 5, p. 416-424, 2017. 
BERNARDO, S.; AMARAL, T. F. Coexistência da desnutrição com a sarcopenia em idosos do conselho de Paços de Ferreiro. Acta Portuguesa de Nutrição, v. 5, p. 1-15, 2016.

BIANCHI, V. E. Role of nutrition on anemia in elderly. Clinical Nutrition Espen, p. 1-11, 2015. DOI: 10.1016/j.clnesp.2015.09.003

BLACKBURN, G. L.; THORNTON, P. A. Nutritional assessment of the hospitalized patient. Medical Clinics of North America, v. 63, n. 5, p. 11.103-11.115, 1979.

BVS. Biblioteca Virtual em Saúde. Atenção primária em saúde: Mini Exame do Estado Mental - MEEM. Disponível em: https://aps.bvs.br/apps/calculadoras/?page=11. Acesso em: 31 maio 2019.

CARDOZO, N. R. et al. Estado nutricional de idosos atendidos por unidades de saúde da família na cidade de Pelotas-RS. Brazilian Society of Parenteral and Enteral Nutrition, v. 32, n. 1, p. 94-98, 2017.

CHUMLEA, W. C. et al. Prediction of body weight for the nonambulatory elderly from anthropometry. Journal of the American Dietetic Association, v. 88, n. 5, p. 564-568, 1988.

CHUMLEA, W. C.; GUO, S. S.; STEINBAUGH, M. L. Prediction of stature from knee height for black and white adults and children with application to mobility-impaired or handicapped persons. Journal of the American Dietetic Association, v. 94, n. 12, p. 1.385-1.390, 1994.

CORRÊA, M. M. et al. Razão cintura-estatura como marcador antropométrico de excesso de peso em idosos brasileiros. Cadernos de Saúde Pública, v. 33, n. 5, e. 00195315, 2017.

DAMO, C. C. et al. Risco de desnutrição e os fatores associados em idosos institucionalizados. Revista Brasileira de Geriatria e Gerontologia, v. 21, n. 6, p. 735-742, 2018.

FRISANCHO, A. R. New norms of upper limb fat and muscle areas for assessment of nutritional status. The American Journal of Clinical Nutrition, v. 34, n. 11, p. 2.540-2.545, 1981.

FRISANCHO, A. R. Anthropometric standards for the assessment of growth and nutritional status. Ann Arbor, MI: The University of Michigan Press, 1990.

GOULART, L. S. et al. Avaliação do estado nutricional associado ao estágio de comprometimento cognitivo em pacientes com demências de um ambulatório de neurologia. Pan American Journal of Aging Research, v. 5, n. 1, p. 7-15, 2017.

IBGE. Instituto Brasileiro de Geografia e Estatísticas. Agência de Notícias. Número de idosos cresce 18\% em 5 anos e ultrapassa 30 milhões em 2017. 2018. Disponível em: https:// agenciadenoticias.ibge.gov.br/. Acesso em: 8 jul. 2020.

LINI, E. V.; PORTELLA, M. R.; DORING, M. Fatores associados à institucionalização de idosos: estudo caso-controle. Revista Brasileira de Geriatria e Gerontologia, v. 19, n. 6, p. 1.004-1.014, 2016.

LIPSCHITZ, D. A. Screening for nutritional status in the elderly. Prim Care, v. 21, n. 1, p. 55-67, 1994.
LOHMAN, T. G.; ROCHE, A. F.; MARTORELL, R. Anthropometric Standardisation Reference Manual. Champaign, IL: Human Kinetics Books, 1988.

MARTINS, M. V. et al. Consumo alimentar de idosos e sua associação com o estado nutricional. Revista Hospital Universitário, v. 42, n. 2, p. 125-131, 2016.

MELLO, A. C. et al. Consumo alimentar e antropometria relacionados à síndrome de fragilidade em idosos residentes em comunidade de baixa renda de um grande centro urbano. Cadernos de Saúde Pública, v. 33, n. 8, e. 00188815, 2017.

MELLO, F. S.; WAISBERG, J. SILVA, M. L. N. Circunferência da panturrilha associa-se com pior desfecho clínico em idosos internados. Geriatrics, Gerontology and Aging, v. 10, n. 2, p. 80-85, 2016.

OLIVEIRA, V. B. et al. Risco cardiovascular, indicadores antropométricos e mini avaliação nutricional reduzida: associação com índice de massa corporal na avaliação nutricional de idosos. Nutrición Clínica y Dietética Hospitalaria, v. 39, n. 1, p. 69-75, 2019.

PADOVANI, R. M. et al. Dietary reference intakes: aplicabilidade das tabelas em estudos nutricionais. Revista de Nutrição, v. 19, n. 6, p. 741-760, 2006.

PAGOTTO, V. et al. Circunferência da panturrilha: validação clínica para avaliação de massa muscular em idosos. Revista Brasileira de Enfermagem, v. 71, n. 2, p. 343-350, 2018.

PANNU, P. K. et al. The associations of vitamin D status and dietary calcium with the metabolic syndrome: an analysis of the Victorian Health Monitor survey. Public Health Nutrition, v. 20, n. 10, p. 1.785-1.796, 2016.

PEREIRA, H. E. F. et al. Perfil nutricional e dietético de idosos atendidos nas estratégias de saúde da família do norte de Minas Gerais. Revista APS, v. 21, n. 2, p 259-266, 2018.

PEREIRA, R. L. M. R.; SAMPAIO, J. P. M. Estado nutricional e práticas alimentares de idosos do Piauí: dados do Sistema de Vigilância Alimentar e Nutricional - Sisvan Web. Revista Eletrônica de Comunicação, Informação \& Inovação em Saúde, v. 13, n. 4, p. 854-862, 2019.

PREVIDELLI, A. N.; GOULART, R. M. M.; AQUINO, R. C. Balanço de macronutrientes na dieta de idosos brasileiros: análises da Pesquisa Nacional de Alimentação 2008-2009. Revista Brasileira de Epidemiologia, v. 20, n. 1, p. 70-80, 2017. RAMÍREZ, G. R. et al. Vitamin E deficiency and oxidative status are associated with prediabetes in apparently healthy subjects. Archives of Medical Research, v. 48, n. 3, p. 257262, 2017.

REJÓN, A. I. R.; LÓPEZ, M. D. R.; ARTACHO, A. Dietary intake and associated factors in long-term care homes in Southeast Spain. Nutrients, v. 11, n. 266, p. 2-14, 2019.

SAARELA, R. K. T. et al. Dentition, nutritional status and adequacy of dietary intake among older residents in assisted living facilities. Gerodontology, v. 33, p. 225-232, 2016.

SALGUEIRO, M. M. H. A. O. et al. Avaliação do estado nutricional e composição corporal de idosos de Embú-Guaçu-SP. Revista Brasileira de Obesidade, Nutrição e Emagrecimento, v. 12, n. 72. p. 446-455, 2018. 
SANTOS, A. S. et al. Estudo de base populacional: perfil sociodemográfico e de saúde em idosos. Revista de Enfermagem Uerj, v. 26, e. 21473, 2018.

SBC. Sociedade Brasileira de Cardiologia. In: FALUDI, A. A. et al. Atualização da Diretriz Brasileira de Dislipidemias e Prevenção da Aterosclerose. Arquivo Brasileiro de Cardiologia, v. 109, n. 5, supl. 1, p. 1-76, 2017.

SCHMIDT, L. et al. Avaliação nutricional de idosos institucionalizados de uma ILPI do interior do Estado do Rio Grande do Sul. Revista Brasileira de Ciências do Envelhecimento Humano, v. 14, n. 1, p. 83-92, 2017.

SERGI, G. et al. Taste loss in the elderly: possible implications for dietary habits. Critical Reviews in Food Science and Nutrition, v. 57, v. 17, p. 3.684-3.689, 2017.

SILVA, J. L. et al. Fatores que reduzem o consumo alimentar de idosos residentes em instituições geriátricas. Unesc em Revista, v. 2, p. 45-56, 2019a.

SILVA, L. M. L. et al. Disfagia e sua relação com o estado nutricional e ingestão calórico-proteica em idosos. Revista Cefac, v. 21, n. 3, e. 15618, 2019b.

SILVA, M. F. et al. Prevalência de constipação intestinal, oferta de fibras alimentares e ingestão hídrica em idosos de uma instituição de longa permanência na cidade de Sete Lagoas, MG. Brazilian Society of Parenteral and Enteral Nutrition, v. 31, n. 3, p. 247-251, 2016.

SILVEIRA, E. A. et al. Obesidade em idosos e sua associação com consumo alimentar, diabetes mellitus e infarto agudo do miocárdio. Arquivos Brasileiros de Cardiologia, v. 107, n. 6, p. 509-517, 2016.

SILVÉRIO, J. K. A. et al. Estado nutricional de idosos institucionalizados: uma revisão de literatura. Visão Acadêmica, v. 17, n. 3, 2016.

TIELAND, M. et al. An even distribution of protein intake daily promotes protein adequacy but does not influence nutritional status in institutionalized elderly. Jamda, p. 1-7, 2017.

VAGETTI, G. C. et al. Associação do índice de massa corporal com a aptidão funcional de idosas participantes de um programa de atividade física. Revista Brasileira de Geriatria e Gerontologia, v. 20, n. 2, p. 216-227, 2017.

VERREIJEN, A. M. et al. A high whey protein -, leucine -, and vitamin $D$ - enriched supplement preserves muscle mass during intentional weight loss in obese older adults: a double-blind randomized controlled trial. The American Journal of Clinical Nutrition, v. 101, p. 279-286, 2015.

WHO. World Health Organization. Physical status: the use and interpretation of anthropometry, 1995.

WHO. World Health Organization. Obesity: preventing and managing the global epidemic, 1998. 